\title{
Anti-tumor effects of bevacizumab in combination with paclitaxel on head and neck squamous cell carcinoma
}

\author{
KYOKO FUJITA, DAISUKE SANO, MACHIKO KIMURA, YUKIKO YAMASHITA, MARIKO KAWAKAMI, \\ YUKARI ISHIGURO, GOSHI NISHIMURA, HIDEKI MATSUDA and MAMORU TSUKUDA
}

Department of Biology and Function in Head and Neck, Yokohama City University Graduate

School of Medicine, 3-9 Fukuura, Kanazawa-ku, Yokohama 236-0004, Japan

Received January 2, 2007; Accepted March 26, 2007

\begin{abstract}
Human tumors are dependent on angiogenesis for growth, and the vascular endothelial growth factor (VEGF) is a major regulator of this process. Bevacizumab (Avastin ${ }^{\circledR}$ ), a monoclonal antibody directed against VEGF, has shown promise in treating a variety of cancers. In this study, we first examined the anti-tumor effects of bevacizumab on head and neck squamous cell carcinoma (HNSCC). Then we examined the effects of bevacizumab combined with paclitaxel, a chemotherapeutic agent, in HNSCC. This is the first demonstration of the anti-tumor effects of bevacizumab on HNSCC. In vitro, bevacizumab did not show any antiproliferative effects against the HNSCC cell lines. However, in vivo, bevacizumab showed dramatic anti-tumor effects against HNSCC tumor xenografts in mice. In addition, treatment with a bevacizumab-paclitaxel combination resulted in a remarkable inhibition of the HNSCC tumor xenografts, compared to the effects of each agent separately. A decreased blood vessel density and an increased apoptotic index were seen in the shrunken tumors. These results suggest that bevacizumab in combination with paclitaxel could have useful clinical application in HNSCC.
\end{abstract}

\section{Introduction}

Angiogenesis, a development of the vascular system from the endothelial cells, is essential during embryogenesis and occurs directly after the process of vasculogenesis. Angiogenesis also plays a pivotal role in several pathological disorders, particularly in tumorigenesis and metastases (1). One of the most potent positive regulators of angiogenesis is the vascular endothelial growth factor (VEGF) (2). VEGF has been identified as a crucial regulator of normal and pathological

Correspondence to: Dr K. Fujita, Department of Biology and Function in Head and Neck, Yokohama City University Graduate School of Medicine, 3-9 Fukuura, Kanazawa-ku, Yokohama 2360004, Japan

E-mail: t056043b@yokohama-cu.ac.jp

Key words: bevacizumab, paclitaxel, head and neck squamous cell carcinoma, antiangiogenesis angiogenesis, with an increased expression being observed in many human tumor types (3). Head and neck cancer cells have been shown to express VEGF $(4,5)$.

Bevacizumab (Avastin) is an anti-VEGF recombinant humanized monoclonal antibody (rhuMAb VEGF). It contains the human immunoglobulin G1 framework (93\%) and murine VEGF-binding complementary-determining regions (7\%) which block the binding of all VEGF-A isoforms to the receptors, inhibiting the biological activity of VEGF (6-8). Bevacizumab has shown promising preclinical and clinical activity against metastatic colorectal cancer $(9,10)$, non-small cell lung cancer (NSCLC) (11), and breast cancer (12). However, bevacizumab alone has only a modest anti-tumor effect. Randomized trials in metastatic colorectal cancer and NSCLC patients have shown that bevacizumab combined with chemotherapy improves the response rate, progression-free survival (PFS), and the overall survival relative to chemotherapy alone (13). Bevacizumab is licensed for use in combination with fluorouracil-based chemotherapy for the first-line treatment of patients with metastatic colorectal cancer in the United States and Europe (9). Recently, the clinical use of bevacizumab combined with paclitaxel in the treatment of advanced NSCLC has shown exciting results $(13,14)$.

This study is the first report on the anti-tumor effects of bevacizumab on head and neck squamous cell carcinoma (HNSCC). In an effort to develop more effective treatment modalities for HNSCC, we examined the effects of bevacizumab plus paclitaxel on HNSCC xenografts in mice. Paclitaxel, a diterpene originally extracted from the bark of the Western yew tree, preferentially binds to the $B$ subunit of tubulin, preventing depolymerization and arresting the cell cycle $(15,16)$. In certain systems, paclitaxel alone has been postulated to have antiangiogenic effects in addition to a direct effect on tumor cells $(15,17-19)$. Paclitaxel has been used alone or in combination with radiation or other chemotherapeutic agents in clinical trials and has demonstrated high response rates in patients with HNSCC (20-22).

In the present study, we investigated the anti-tumor activity of bevacizumab in combination with paclitaxel and their anti-tumor mechanisms.

\section{Materials and methods}

Drugs. Bevacizumab (Avastin, Genentech, South San Francisco, CA) was purchased. For the antiproliferative assay, 
a stock solution was made with $100 \%$ dimethyl sulfoxide (DMSO) and diluted with culture media. For a study of the anti-tumor activity, bevacizumab was dissolved in saline, and paclitaxel was dissolved in absolute ethanol, cremophor ELP according to a previously reported procedure $(23,24)$. Dosing preparations of both agents were prepared on the day of use.

Cell lines and culture conditions. Human HNSCC cell lines were examined in this study. The origins of these cell lines were the hypopharynx (YCU-H891), mesopharynx (YCUM862, KCC-M871, YCU-M911), larynx (KCC-L871, YCUL891), tongue (KCC-T871, KCC-T873), and the maxillary sinus (KCC-MS871, YCU-MS861). These cell lines were maintained in RPMI-1640 medium (Life Technologies Inc., Tokyo, Japan) supplemented with $10 \%$ fetal bovine serum, $2 \mathrm{mmol} / \mathrm{l} \mathrm{L}$-glutamine, $100 \mathrm{units} / \mathrm{ml}$ penicillin, and $100 \mu \mathrm{g} /$ $\mathrm{ml}$ streptomycin. The cells were incubated at $37^{\circ} \mathrm{C}$ in an atmosphere containing $5 \% \mathrm{CO}_{2}$.

In vitro proliferation assays. MTT assay was performed using ten human HNSCC cell lines. These cells were plated in 96-well U-bottomed plates (Falcon; Becton-Dickinson Labware, Lincoln Park, NJ, USA) at a concentration of $5 \times 10^{3}$ cells/well. The plates were allowed to incubate for $24 \mathrm{~h}$ prior to the drug treatment. After 48-h exposure to the drugs, the MTT assay was carried out with Tetra Color One (Seigaku Co., Ltd. Tokyo, Japan). The proliferation experiments were performed with and without stimulation by the addition of bevacizumab and paclitaxel.

In vivo anti-tumor activity studies. Female BALB/c $n u / n u$ nude mice, 6 weeks old, were obtained from Oriental Yeast, Tokyo, Japan. The mice were maintained in a laminar flow room with a constant temperature and humidity. Suspensions of YCU-H891 cells (100 $\mu 1$ ) (final concentration, $1 \times 10^{7}$ cells $/ \mathrm{ml}$ ) were injected s.c. into the right flank of the mice. Tumor-bearing mice were randomized $(n=7)$ when the mean tumor volume was $50-100 \mathrm{~mm}^{3}$. Each group was closely matched before treatment, which began one week after cell transplantation. The mice were treated i.p. with bevacizumab ( $2 \mathrm{mg} / \mathrm{kg} /$ day or $4 \mathrm{mg} / \mathrm{kg} /$ day on days 1 and 4 of each week for 4 weeks). Paclitaxel was administered i.p. (10 mg/kg/day on days 8 and 15). In combination studies, groups of tumorbearing mice were treated with both bevacizumab $(2 \mathrm{mg} / \mathrm{kg} /$ day or $4 \mathrm{mg} / \mathrm{kg} /$ day, i.p.) and paclitaxel $(10 \mathrm{mg} / \mathrm{kg} / \mathrm{day}$, i.p.). The control mice were administered the solvent solution. Tumor diameters in the control and treated groups were measured weekly with a Vernier caliper. Tumor volume (V) was determined by the equation: $\mathrm{V}=\mathrm{ab}^{2} / 2$ ( $\mathrm{a}=$ length; $\mathrm{b}=$ width).

Evaluation of vessel density. In order to estimate the vessel density, the vessels in the tumor tissues were stained with an anti-von Willebrand Factor (vWF) antibody (1:200; DAKO, Denmark). The tumors were excised and blocked with $2 \%$ goat serum and $1 \%$ bovine serum albumin in PBS and stained with the antibody. Slides were developed using 3, 3'diaminobenzidine substrate biotinylated peroxidase reagent (Vector Laboratories, Inc., Burlingame, CA, USA). Vessel

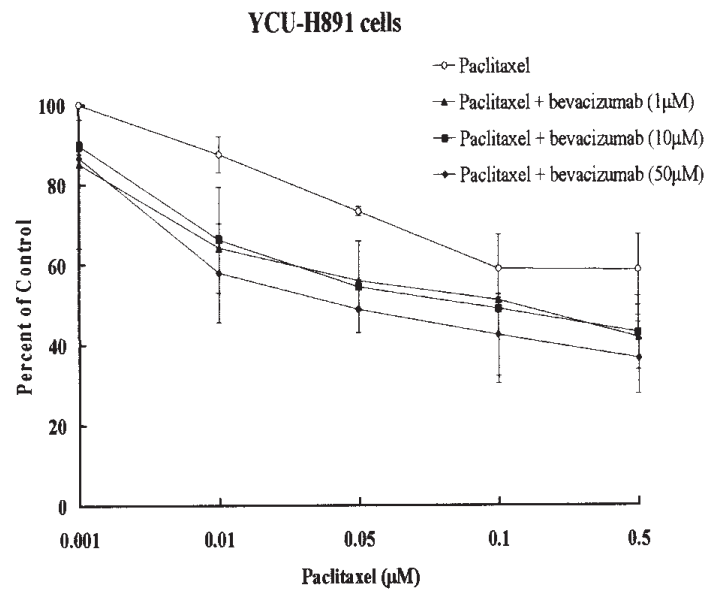

Figure 1. Growth inhibitory effect of treatment with paclitaxel in combination with bevacizumab on the YCU-H891 head and neck squamous cell carcinoma (HNSCC) cell lines. No significant antiproliferative effect of paclitaxel with bevacizumab was observed in the HNSCC cells. The error bars indicate SE.

density was determined by counting the stained vessels in the fields at x200 magnification. Five fields per histological section were included in the analysis.

Evaluation of apoptosis. Terminal deoxynucleotidyl transferase-mediated cUDP nick end-labeling (TUNEL) was done for the evaluation of apoptosis. This was evaluated using an Apoptosis In Situ Detection Kit (Wako Chemical, Osaka, Japan). The apoptotic index was calculated as the percentage of positive cell nuclei stained with peroxidase relative to the total number of cells from a minimum of five microscopic fields from each section.

Statistical analysis. For the statistical analyses of the vessel density, apoptotic index, and the in vivo anti-tumor activity, we used the Student's paired t-test. A value of $\mathrm{P}<0.05$ was considered significant.

\section{Results}

Antiproliferative effects of bevacizumab and paclitaxel on HNSCC cells. We first determined the antiproliferative effects of different concentrations of bevacizumab alone and/or in combination with the indicated concentration of paclitaxel on the HNSCC cell lines. Bevacizumab alone had no activity as a single agent in the proliferation assay (data not shown). Next, a series of experiments were done in order to evaluate the antiproliferative effects of treatment with a bevacizumab-paclitaxel combination on the HNSCC cell line. As illustrated in Fig. 1, no significant synergistic growth inhibitory effect was observed at any of the doses.

In vivo study of the anti-tumor effects of bevacizumab in combination with paclitaxel on HNSCC xenografts. We investigated the in vivo anti-tumor activity of bevacizumab and/or paclitaxel in nude mice bearing HNSCC xenografts. HNSCC tumor growth was inhibited by bevacizumab in vivo. The bevacizumab treatment $(2-4 \mathrm{mg} / \mathrm{kg} /$ day $)$ of nude mice bearing established HNSCC tumor xenografts produced a 
$\mathbf{A}$

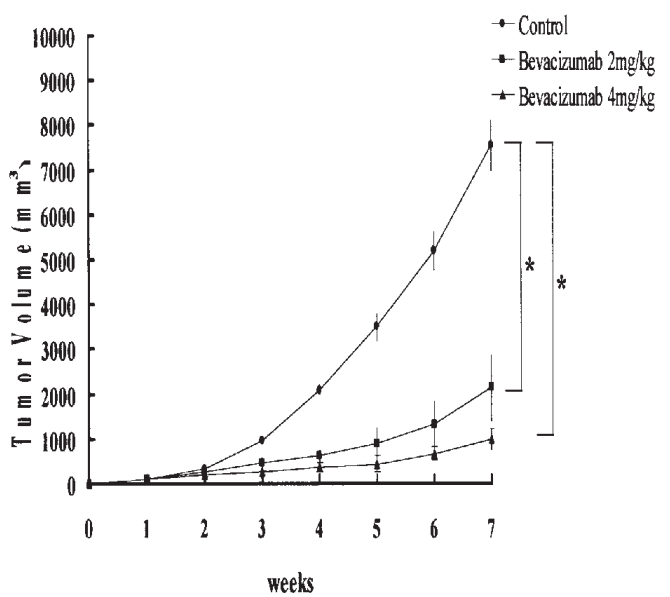

B

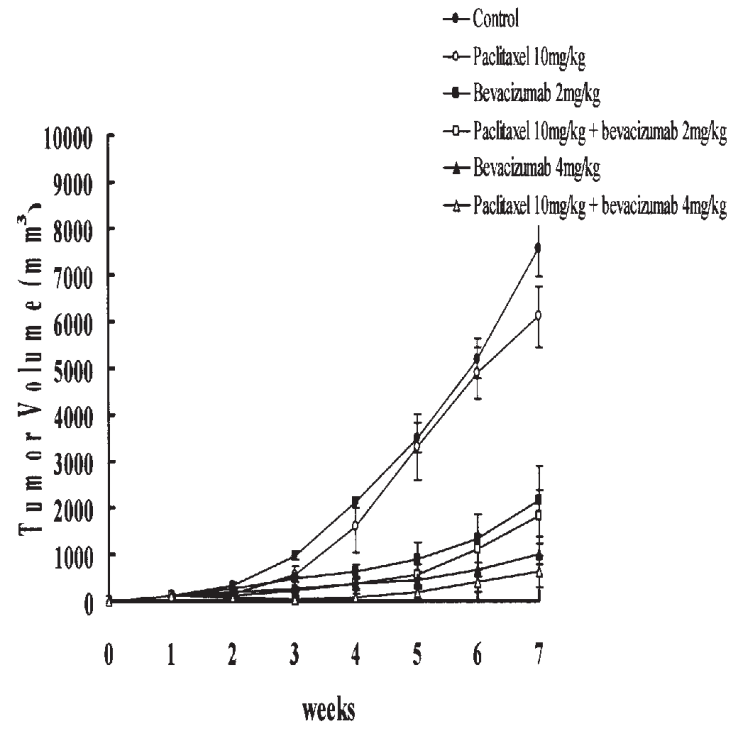

Figure 2. Effects of bevacizumab and paclitaxel on the growth of the head and neck squamous cell carcinoma xenograft model. $\bullet$, Tumor treated with the vehicle (1\% polysorbate in deionized water). (A) Bevacizumab ( $\mathbf{\square}, 2 \mathrm{mg} / \mathrm{kg}$ i.p.; $\Delta, 4 \mathrm{mg} / \mathrm{kg}$ i.p.) administered twice weekly demonstrated significant growth inhibition $(\mathrm{P}<0.05)$. (B) Paclitaxel (o, $10 \mathrm{mg} / \mathrm{kg}$ i.p. on days 8 and 15) and bevacizumab (๘,2 mg/kg i.p.; $\mathbf{\Lambda}, 4 \mathrm{mg} / \mathrm{kg}$ i.p.) were administered for the duration of the study. Paclitaxel with bevacizumab ( $\neg$, paclitaxel i.p. + bevacizumab $2 \mathrm{mg} / \mathrm{kg}$ i.p.; $\Delta$, paclitaxel i.p + bevacizumab $4 \mathrm{mg} / \mathrm{kg}$ i.p) resulted in greater growth inhibition $(\mathrm{P}<0.05)$. Points, mean tumor volume $\left(\mathrm{mm}^{3}\right)$; bar, $\mathrm{SE}$ ( $\mathrm{n}=7$ mice per group); error bars indicate $\mathrm{SE} ;{ }^{*} \mathrm{P}<0.05$ vs control.

dose-dependent inhibition of tumor growth (Fig. 2A). As shown in Fig. 2B, the paclitaxel $(10 \mathrm{mg} / \mathrm{kg} /$ day $)$ treatment alone did not cause a complete regression of the tumors, but the combined treatment with bevacizumab plus paclitaxel in the present model resulted in a marked reduction in tumor volume compared to the control group $(\mathrm{P}<0.05$; Fig. $2 \mathrm{~B})$. The degree of inhibition observed in the combination treatment was greater than that observed with either agent alone at the end of the treatment period (mean tumor volumes, $6100 \mathrm{~mm}^{3}$ with paclitaxel, $2137 \mathrm{~mm}^{3}$ with bevacizumab at $2 \mathrm{mg} / \mathrm{kg} /$ day, $1002 \mathrm{~mm}^{3}$ with bevacizumab at $4 \mathrm{mg} / \mathrm{kg} / \mathrm{day}$, $1800 \mathrm{~mm}^{3}$ with paclitaxel plus bevacizumab at $2 \mathrm{mg} / \mathrm{kg} /$ day, $620 \mathrm{~mm}^{3}$ with paclitaxel and bevacizumab at $4 \mathrm{mg} / \mathrm{kg}$ day; $\mathrm{P}<0.05$ vs controls).

Effects of combination treatment on microvascular content in an established HNSCC xenograft. The vessel density of the
A

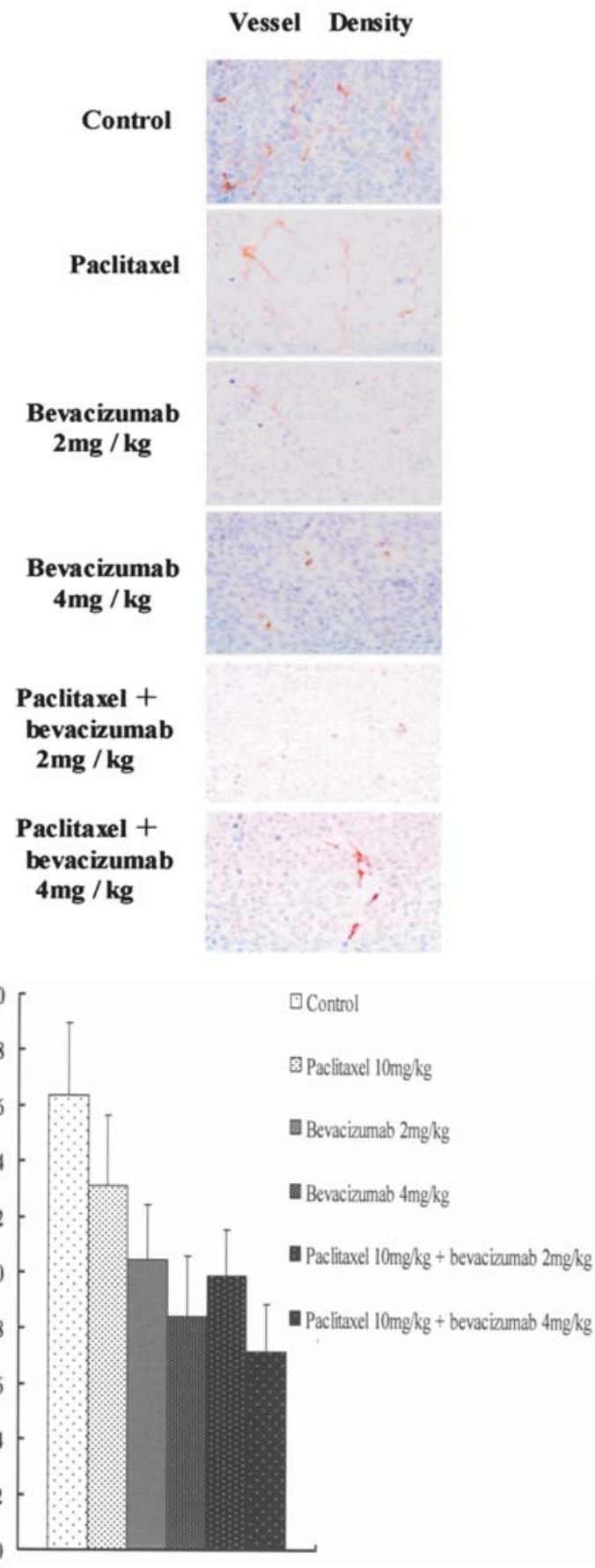

Figure 3. Vessel density in YCU-H891 tumor xenografts. (A) Vascular staining. The changes in vessel density of the tumor cells stained with an anti-von Willebrand Factor antibody (x400). (B) Effects of bevacizumab and paclitaxel on the vessel density. Data represent mean values $( \pm S D)$. Significant difference by Student's t-test. ( ${ }^{*} \mathrm{p}<0.05$ vs control).

xenograft tumors was examined in order to clarify the antitumor mechanisms. Fig. 3 shows the results of the microvessel density, using a monoclonal antibody against vWF. The number of microvessels positive for anti-vWF in the group treated with bevacizumab alone (at $4 \mathrm{mg} / \mathrm{kg}$ ) and bevacizumab plus paclitaxel was significantly lower than that of the control group.

Effects of combination treatment on apoptosis in an established HNSCC xenograft. A histopathological analysis of the xenograft samples was done in order to examine the 
A

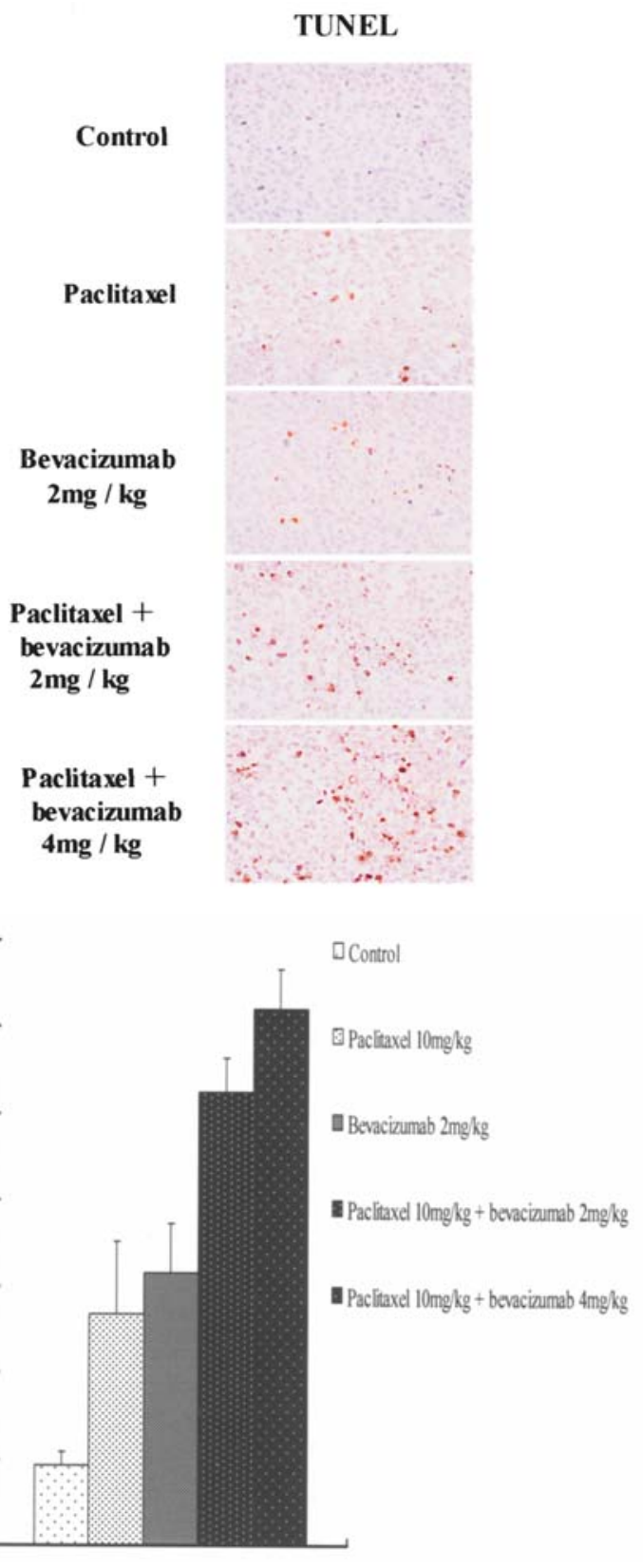

Figure 4. Apoptotic events in YCU-H891 tumor xenografts. (A) Terminal deoxynucleotidyl transferase-mediated cUDP nick end-labeling (TUNEL) staining. Histopathological analysis of apoptosis by TUNEL (x400). (B) Effects of bevacizumab and paclitaxel on the apoptotic index (TUNEL). Data represent mean values $( \pm \mathrm{SD})$. Significant difference by the Student's ttest. $\left({ }^{*} \mathrm{p}<0.01\right.$ vs control).

apoptotic events. Fig. 4 shows the results of the TUNEL staining. There was a significant difference between the control group and the bevacizumab plus paclitaxel group. The combination treatment resulted in an increased number of apoptotic cells (TUNEL-positive cells). Due to the insufficient TUNEL staining, it was not possible to obtain a meaningful statistical analysis of TUNEL staining of the tumors treated with bevacizumab alone at $4 \mathrm{mg} / \mathrm{kg}$.

\section{Discussion}

In the present study, the treatment with bevacizumab alone, or with bevacizumab plus paclitaxel did not inhibit the growth of HNSCC cells in vitro. However, bevacizumab in combination with paclitaxel produced greater inhibition than either agent alone. We also examined the mechanisms of the combination therapy with the histopathological method.

Bevacizumab had no inhibitory effect on HNSCC in vitro. It has generally been found that bevacizumab does not inhibit tumor cell growth $(13,25)$. The inhibition of capillary formation may be required in order to obtain antiproliferative effects. Kim et al (7) have shown that the treatment of tumor cell lines with bevacizumab has no effect on their proliferation rate. These reports support our results. The inhibition of vascular endothelial cell proliferation or morphogenesis results in a characteristic decrease in tumor capillary density (26).

In this study, bevacizumab alone significantly inhibited tumor growth. However, Fox et al (15) have shown that the bevacizumab-mediated growth inhibition no longer occurs after the treatment is discontinued, and the treated tumor begins to grow again. Hu et al (27) have shown that bevacizumab alone does not significantly inhibit tumor growth.

Treatment with bevacizumab plus paclitaxel significantly decreased tumor growth. Therefore, bevacizumab is licensed for use in combination with fluorouracil-based chemotherapy for the first-line treatment of patients with metastatic colorectal cancer in the United States and Europe $(9,28)$. Clinical trials are currently under way with bevacizumab in combination with various chemotherapeutic agents (11). Therefore, we suggest that combination studies should be conducted on HNSCC.

In the present study, the histopathological analysis of the xenograft samples showed that treatment with bevacizumab plus paclitaxel decreased the vessel density and increased apoptosis compared to the single-agent administration. The detailed mechanism of this combination therapy is not clear. However, the anti-tumor activity was confirmed by the histopathological findings. Our results also support the hypothesis that bevacizumab in combination with paclitaxel inhibits new vessel formation and induces apoptosis in our HNSCC tumor model. Paclitaxel binds to tubulin, retards microtubule depolymerization, impairs mitosis, blocks cell cycle progression, and facilitates apoptosis (19). In addition, paclitaxel has antiangiogenic activity $(15,17,18)$. Thus, tumor growth could be affected not only by direct cytotoxicity but also by the inhibition of new vessel formation. Another study demonstrated that the blocking of VEGF signaling can significantly enhance the efficacy of chemotherapeutic agents (29).

There are certain hypotheses regarding the feasibility of combining bevacizumab with chemotherapy. Jain (30) proposed that if tumor vasculature could be 'normalized' with bevacizumab therapy, it would facilitate a better delivery of therapeutic agents to the tumor. In addition to its direct antiangiogenic effects, bevacizumab can also improve the delivery of chemotherapeutic agents by altering tumor vasculature and decreasing the elevated interstitial pressure in tumors $(9,30,31)$. Another hypothesis is based on the fact that cell stress response up-regulates VEGF production. The administration of bevacizumab can block the cell stress response induced by paclitaxel, thus resulting in an increased inhibition of tumor growth by the combination of these two agents (32).

Griffon-Etienne et al (33) have shown that paclitaxel has antiangiogenic effects that are increased when lower doses of paclitaxel are given more frequently. Klement et al (34) 
suggested that low-dose chemotherapy has proapoptotic effects against endothelial cells. Therefore, simultaneously targeting VEGF could amplify the anti-tumor effects of chemotherapy. Paclitaxel has been very beneficial in clinical trials of HNSCC; e.g., it has improved the response rate of the primary tumor and the elongation of median survival time $(20,21)$. However, in many patients, adverse effects have been observed $(20,35)$. In addition, using antiangiogenic agents acting through VEGF inhibition is an attractive therapeutic strategy as this could be less toxic than conventional cytotoxic therapy. The addition of bevacizumab could reduce the doses of paclitaxel, thereby reducing toxicity.

In conclusion, our results demonstrate that bevacizumab in combination with paclitaxel show greater inhibition than either agent alone on the HNSCC xenografts in a mouse model. Our data also demonstrate the anti-tumor mechanisms of the inhibition of angiogenesis and the induction of apoptosis. This study provides an experimental basis for the clinical development of therapy based on the combination of bevacizumab with paclitaxel in HNSCC patients.

\section{References}

1. Kerbel RS: Tumor angiogenesis: past, present and the near future. Carcinogenesis 21: 505-515, 2000.

2. Ferrara N: Vascular endothelial growth factor: basic science and clinical progress. Endocr Rev 25: 581-611, 2004.

3. Ferrara $\mathrm{N}$ and Davis-Smyth $\mathrm{T}$ : The biology of vascular endothelial growth factor. Endocr Rev 18: 4-25, 1997.

4. Borgstrom P, Bourdon MA, Hillan KJ, Sriramarao P and Ferrara N: Neutralizing anti-vascular endothelial growth factor antibody completely inhibits angiogenesis and growth of human prostate carcinoma micro tumors in vivo. Prostate 35: 1-10, 1998.

5. Berse B, Brown LF, Van de Water L, Dvorak HF and Senger DR: Vascular permeability factor (vascular endothelial growth factor) gene is expressed differentially in normal tissues, macrophages, and tumors. Mol Biol Cell 3: 211-220, 1992.

6. Presta LG, Chen H, O'Connor SJ, et al: Humanization of an anti-vascular endothelial growth factor monoclonal antibody for the therapy of solid tumors and other disorders. Cancer Res 57: 4593-4599, 1997

7. Kim KJ, Li B, Houck K, Winer J and Ferrara N: The vascular endothelial growth factor proteins: identification of biologically relevant regions by neutralizing monoclonal antibodies. Growth Factors 7: 53-64, 1992.

8. Gridelli C, Rossi A and Maione P: New antiangiogenetic agents and non-small cell lung cancer. Crit Rev Oncol Hematol 60: 76-86, 2006.

9. Hurwitz H, Fehrenbacher L, Novotny W, et al: Bevacizumab plus irinotecan, fluorouracil, and leucovorin for metastatic colorectal cancer. N Engl J Med 350: 2335-2342, 2004.

10. Kabbinavar FF, Hambleton J, Mass RD, Hurwitz HI, Bergsland E and Sarkar S: Combined analysis of efficacy: the addition of bevacizumab to fluorouracil/leucovorin improves survival for patients with metastatic colorectal cancer. J Clin Oncol 23: 3706-3712, 2005.

11. Johnson DH, Fehrenbacher L, Novotny WF, et al: Randomized phase II trial comparing bevacizumab plus carboplatin and paclitaxel with carboplatin and paclitaxel alone in previously untreated locally advanced or metastatic non-small-cell lung cancer. J Clin Oncol 22: 2184-2191, 2004.

12. Cobleigh MA, Langmuir VK, Sledge GW, et al: A phase I/II dose-escalation trial of bevacizumab in previously treated metastatic breast cancer. Semin Oncol 30: 117-124, 2003.

13. Sandler AB, Johnson DH and Herbst RS: Anti-vascular endothelial growth factor monoclonals in non-small cell lung cancer. Clin Cancer Res 10: 4258S-4262S, 2004.

14. Sandler A and Herbst R: Combining targeted agents: blocking the epidermal growth factor and vascular endothelial growth factor pathways. Clin Cancer Res 12: 4421S-4425S, 2006.
15. Fox WD, Higgins B, Maiese KM, et al: Antibody to vascular endothelial growth factor slows growth of an androgenindependent xenograft model of prostate cancer. Clin Cancer Res 8: 3226-3231, 2002.

16. Eisenhauer EA and Vermorken JB: The taxoids. Comparative clinical pharmacology and therapeutic potential. Drugs 55: 5-30, 1998.

17. Sweeney CJ, Miller KD, Sissons SE, et al: The antiangiogenic property of docetaxel is synergistic with a recombinant humanized monoclonal antibody against vascular endothelial growth factor or 2-methoxyestradiol but antagonized by endothelial growth factors. Cancer Res 61: 3369-3372, 2001.

18. Belotti D, Vergani V, Drudis T, et al: The microtubule-affecting drug paclitaxel has antiangiogenic activity. Clin Cancer Res 2: 1843-1849, 1996.

19. Lau DH, Xue L, Young LJ, Burke PA and Cheung AT: Paclitaxel (Taxol): an inhibitor of angiogenesis in a highly vascularized transgenic breast cancer. Cancer Biother Radio-pharm 14: 31-36, 1999.

20. Forastiere AA, Shank D, Neuberg D, Taylor SGT, DeConti RC and Adams G: Final report of a phase II evaluation of paclitaxel in patients with advanced squamous cell carcinoma of the head and neck: an Eastern Cooperative Oncology Group trial (PA390). Cancer 82: 2270-2274, 1998.

21. Cortes-Funes $\mathrm{H}$ and Aisner J: Paclitaxel in head and neck cancer and other tumor types: chairmen's introduction. Semin Oncol 24 (Suppl 2): 51-57, 1997.

22. Forastiere AA and Urba SG: Single-agent paclitaxel and paclitaxel plus ifosfamide in the treatment of head and neck cancer. Semin Oncol 22: 24-27, 1995.

23. Cassinelli G, Lanzi C, Supino R, et al: Cellular bases of the antitumor activity of the novel taxane IDN 5109 (BAY59-8862) on hormone-refractory prostate cancer. Clin Cancer Res 8: 2647-2654, 2002.

24. Polizzi D, Pratesi G, Tortoreto M, et al: A novel taxane with improved tolerability and therapeutic activity in a panel of human tumor xenografts. Cancer Res 59: 1036-1040, 1999.

25. Gossmann A, Helbich TH, Kuriyama N, et al: Dynamic contrast-enhanced magnetic resonance imaging as a surrogate marker of tumor response to anti-angiogenic therapy in a xenograft model of glioblastoma multiforme. J Magn Reson Imaging 15: 233-240, 2002.

26. Parangi S, O'Reilly M, Christofori G, et al: Antiangiogenic therapy of transgenic mice impairs de novo tumor growth. Proc Natl Acad Sci USA 93: 2002-2007, 1996.

27. Hu L, Hofmann J, Zaloudek C, Ferrara N, Hamilton T and Jaffe RB: Vascular endothelial growth factor immunoneutralization plus Paclitaxel markedly reduces tumor burden and ascites in athymic mouse model of ovarian cancer. Am J Pathol 161: 1917-1924, 2002.

28. Kuramochi H, Hayashi K, Uchida K, et al: Vascular endothelial growth factor messenger RNA expression level is preserved in liver metastases compared with corresponding primary colorectal cancer. Clin Cancer Res 12: 29-33, 2006.

29. Inoue K, Slaton JW, Davis DW, et al: Treatment of human metastatic transitional cell carcinoma of the bladder in a murine model with the anti-vascular endothelial growth factor receptor monoclonal antibody DC101 and paclitaxel. Clin Cancer Res 6: 2635-2643, 2000.

30. Jain RK: Normalizing tumor vasculature with anti-angiogenic therapy: a new paradigm for combination therapy. Nat Med 7: 987-989, 2001.

31. Willett CG, Boucher Y, di Tomaso E, et al: Direct evidence that the VEGF-specific antibody bevacizumab has antivascular effects in human rectal cancer. Nat Med 10: 145-147, 2004.

32. Yang JC: Bevacizumab for patients with metastatic renal cancer: an update. Clin Cancer Res 10: 6367S-6370S, 2004.

33. Griffon-Etienne G, Boucher Y, Brekken C, Suit HD and Jain RK: Taxane-induced apoptosis decompresses blood vessels and lowers interstitial fluid pressure in solid tumors: clinical implications. Cancer Res 59: 3776-3782, 1999.

34. Klement G, Baruchel S, Rak J, et al: Continuous low-dose therapy with vinblastine and VEGF receptor-2 antibody induces sustained tumor regression without overt toxicity. J Clin Invest 105: R15-R24, 2000.

35. Jaglowski JR and Stack BC Jr: Enhanced growth inhibition of squamous cell carcinoma of the head and neck by combination therapy of fusaric acid and paclitaxel or carboplatin. Cancer Lett 243: 58-63, 2006. 